\title{
Physics of soil erosion at the microscale
}

\author{
Pierre Philippe ${ }^{1, \star}$, Pablo Cuéllar ${ }^{1,2}$, Florian Brunier-Coulin ${ }^{1}$, Li-Hua Luu $^{1}$, Nadia Benahmed $^{1}$, Stéphane Bonelli ${ }^{1}$, and \\ Jean-Yves Delenne ${ }^{3}$ \\ ${ }^{1}$ IRSTEA, UR RECOVER, 3275 route de Cézanne, 13182 Aix-en-Provence, France \\ ${ }^{2}$ BAM, Division 7.2 Buildings and Structures, Unter den Eichen 87, 12205 Berlin, Germany \\ ${ }^{3}$ UMR IATE, INRA/CIRAD/Montpellier SupAgro, 2 place Pierre Viala, 34060 Montpellier, France
}

\begin{abstract}
We focus here on the major and still relevant issue of soil erosion by fluid flows, and more specifically on the determination of both a critical threshold for erosion occurrence and a kinetics that specifies the rate of eroded matter entrainment. A state-of-the-art is first proposed with a critical view on the most commonly used methods and erosion models. It is then discussed an alternative strategy, promoting the use of model materials that allow systematic parametric investigations with the purpose of identifying more precisely the local mechanisms responsible for soil particle erosion and ultimately quantifying both critical onsets and kinetics, possibly through existing or novel empirical erosion laws. Finally, we present and discuss several examples following this methodology, implemented either by means of experiments or numerical simulations, and coupling erosion tests in several particular hydrodynamical configurations with wisely selected mechanical tests.
\end{abstract}

\section{Introduction}

Erosion and transport of cohesive soil sediments by fluid flows are of primary concern in many processes, either in natural or manmade environments, as fluvial morphological changes, spreading of pollutant or radionucleides in river sediments, impact of agricultural soil erosion on the global carbon cycle, local scour at bridge piers, external and internal erosion of earthen hydraulic structures [1].

The present contribution focuses on two related fundamental issues: (i) How to properly predict a critical condition, if any, for occurrence of erosion? (ii) How to accurately quantify the amount of eroded soil particles? It is first presented here a brief synthesis of the most usual methods used to tackle these issues with particular attention to what are the major remaining problems or contradictions which could limit the general scope, or even the validity, of these previous approaches. As a consequence, an alternative methodology is proposed, based on systematic investigations, either experimental or by means of numerical models, with artificial cohesive materials and aims to combine erosion tests in some specific fluid flow configurations with better targeted, or even specially developed, mechanical characterisations of our model systems. As illustrated by several preliminary tests following the previous strategy, a specific scope is put on the local processes of matter entrainment by a fluid flow inducing what is more globally called soil's erosion at a more wider scale, both in space and time.

\footnotetext{
^e-mail: pierre.philippe@irstea.fr
}

\section{Quantification of soil erosion and related issues}

Being able to quantify erosion of a soil by a neighboring fluid flow requires first to select both space and time scales over which the amount of eroded matter and the hydrodynamical load exerted by the fluid flow should be considered and quantified. Owing to the large variety of practical issues, the space scale can span from kilometer squares to microns, including for instance watersheds, riverbanks, soil specimens in the lab, or only few sediment layers, while the duration under consideration can go from seconds to months, or even more in some geological phenomena. This first choice prescribes the type of erosion models needed to achieve a consistent prediction, in particular regarding the quantities that can be used to account for the action of the eroding fluid flow. In a soil mechanics perspective as will be considered in the following, the size of a soil sample under study is typically equal to some centimeters, or tens of centimeters, and the rates of erosion are estimated over time periods of seconds to minutes. Regarding the flow regime which is turbulent in most applications, an hydrodynamical indicator, able to account for the hydrodynamic force exerted by the flow on the soil surface, is not easy to quantify since the fluid flow highly fluctuates in time and also, most often, in space. The simplest and most commonly followed solution consists in a crude averaging of the chosen quantity. Alternative approaches can be developed, mostly based on statistical modeling, but might be hard to apply successfully in practice since a realistic probability density function is needed. The same uncertainty about the use of average 
quantities arises considering the mechanical resistance of a soil to erosion. Here again, it is likely that the sensitivity to erosion varies from location to location within a soil, reflecting somehow the inherent heterogeneous nature of the material. Note also that in the particular case of a sandy soil, following the pioneer studies by Shields on fluvial sediment transport, the resistance to erosion can be expressed through a critical dimensionless shear-stress whose value can be approximated from simplified mechanistic models describing the equilibrium of a single particle and accounting for drag force, buoyant weight and friction. The more usual situation of a cohesive soil is far much complicated and knowledge is lacking on local processes of erosion at a natural soil surface to consider developing similar mechanistic models.

In practice, the very large majority of previous and actual studies seeking to assess the sensitivity of natural soils to fluid flow erosion have based their analysis assuming the following expression of a local erosion law that accounts for a threshold and relates $\dot{m}$, the rate of mass removal by units of time and surface, to $\tau$, the shear-stress exerted by the fluid flow at the soil's surface $[2,4]$ :

$$
\begin{gathered}
\dot{m}=k_{e r}\left(\tau-\tau_{c}\right) \text { if } \tau>\tau_{c}, \\
\dot{m}=0 \text { otherwise. }
\end{gathered}
$$

In this purely empirical modeling, $\tau_{c}$ (in $P a$ ) is a critical shear-stress and $k_{e r}$ (in $s . m^{-1}$ ) is a kinetic erosion coefficient. Both parameters are sometimes called soil's erodibility. Several erosion tests have been developed to provide direct measurements or (sometimes crude) estimates of eroded mass and hydrodynamical shear-stress. Based on the local erosion law in Eq. 1 (or a slightly modified version), a fitting procedure ultimately provides the erodibility parameters. Correlations between those parameters and more common soil's properties are still being explored. If rather accurate trends are observed for a same soil, very different and hardly predictable values of erodibility can be found from one soil to another and also possibly from one erosion device to another. The latter result raises in particular questions regarding the postulated intrinsic nature of erodibility. The many different, and sometimes contradictory, empirical relationships proposed in the literature are far from providing a definitive answer. Another general issue concerns the use of average quantities, especially when describing the fluid flow, which appears in serious contradiction with a process as erosion which assumes the existence of an inherent soil's threshold. Indeed, the local hydrodynamic stress values are naturally distributed (fluctuations, turbulence) and the highest portion of this distribution might exceed the soil's critical threshold $\tau_{c}$ while the average stress remains below it, generating intermittent particle extraction and consequently sustaining over time a non-zero rate of erosion.

\section{Methodology}

Regarding the related literature, very little is known about the real processes at work at particle scale and

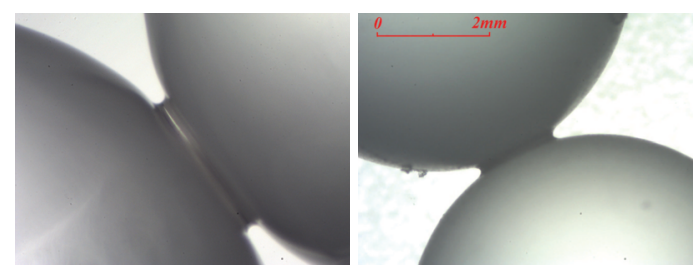

Figure 1. (Left) Capillary bridge of Ucon Oil between $3 \mathrm{~mm}$ glass beads. (Right) Solid bridge of paraffin between $7 \mathrm{~mm}$ glass beads after solidification of the original capillary bridge of liquid paraffin.

which commonly hide behind the general term of erosion. Furthermore, as discussed previously, no clear picture has emerged from the most popular route based on direct empirical correlations between resistance to erosion and many different usual material properties obtained for a large variety of natural soils. This approach possibly reaches its limits, facing a rather complex phenomenon and the present contribution proposes to reverse the methodology by using purely artificial materials which can advantageously allow for both a much more accurate control of some key properties (as cohesion, particle size, etc) and, whenever possible, for a direct visualization of the basic local processes of solid matter removal by a fluid flow. Our goal is first to gain insights from the fundamental scale in order to reach a better understanding of the microstructural scenarios of soil's erosion. Second, to identify and rationalize these local mechanisms would constitute an important step towards clearer relationships between some pertinent usual properties of a material, whose characterization might requires the development of specific mechanical tests, and some more application-oriented empirical characterizations as the previous erodibility parameters $k_{e r}$ and $\tau_{c}$, or possibly some new ones related to alternative local erosion laws to be proposed. Such a breakthrough could considerably supplement the usual approach for erosion risk assessment and accordingly motivates this work.

\subsection{Model materials}

For the present contribution, three different types of cohesive soils have been considered. The first type of materials that can be rather simply prepared and studied is made of large granular particles with adhesive bonds at inter-particle contacts. Such contact bonds can be either liquid or solid as illustrated in Figure 1. It is to be noticed the main differences between liquid and solid bonds: solid bridges are usually far much resistant than liquid ones but the latter can be broken and then created back with strong hysteresis while the rupture of a solid bond is permanent and irreversible. Regarding the grains, they can come from either a natural sandy material or an artificial one as the previous glass beads in Fig. 1 which can advantageously enable the additional implementation of two coupled optical techniques, Refractive Index Matching and Planar Laser Induced Fluorescence, to access to a direct visual- 


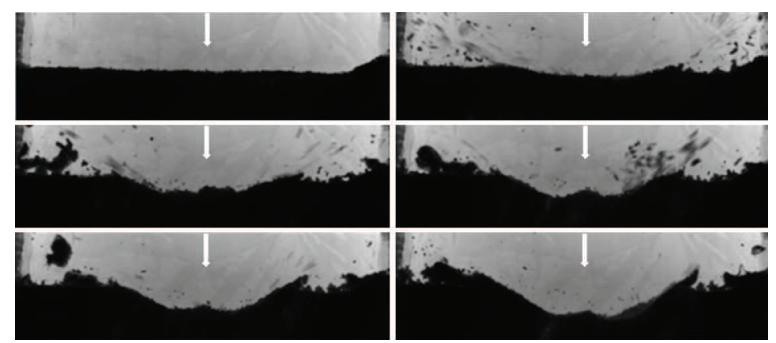

Figure 2. Typical sequence (from left to right and top to bottom) of scour erosion of a magic sand during a $2 \mathrm{D}$ water jet test. The arrow indicates the location of the impinging water jet.

ization of the local erosion process provided that the eroding fluid is an index-matched mixture [5]. The behaviour of such a cohesive granular material with solid bonds can also be investigated by means of the coupling between the Discrete Element Method (DEM) and the Lattice Boltzmann Method (LBM), which is certainly today one of the numerical methods being the most increasingly used for the modeling of fluid-grain systems. An attractive bond at each grain contact is implemented thanks to an additional cohesion model between the solid particles which is based on a relatively straightforward rheology (i.e. viscoelasticperfectly-plastic) and on the de-bonding limit (yield) conditions introduced by Delenne and coworkers [3] which permit the consideration of bond ruptures in tension, shear and bending conditions.

The second type of model cohesive soils that can be easily studied through systematic investigations are chemically-treated sands, as magic sand and kinetic sand. The first one is a building toy made of hydrophobic sand grains and can consequently be viewed as a granular material with an effective "skin" cohesion arising from the Laplace over-pressure at the interface between air and water. Kinetic sand, made of $98 \%$ sand and $2 \%$ polydimethylsiloxane (a type of silicone oil), is another toy material specially developed to mimic the physical behaviour of wet sand with reversible adhesion between grains.

The third kind of artificial cohesive soils corresponds to homogeneous materials, modeled in a continuous medium perspective and able to mimic loamy soils made of very small particles with strong attractive interactions. A synthetic clay as Laponite appears as a sound choice since its yield-stress rheology can be systematically varied and because it also has the great advantage to be transparent and thus to allow here again for direct visualization of solid matter retrieval during an erosion test.

\subsection{Coupling hydrodynamic and mechanical tests}

As an integral part of our methodology, erosion and mechanical tests must be implemented in parallel. The choice of the hydrodynamical configuration inducing erosion determines the ability to measure and/or model accurately some key quantities as shear-stress or velocity and to reach sufficient intensity level for erosion to occur. In the present paper, some already existing configurations, as jet erosion

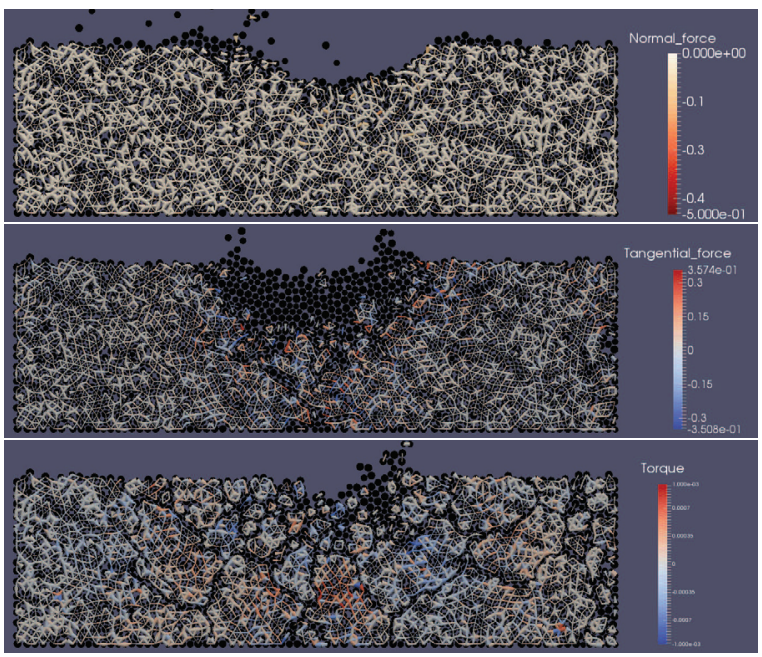

Figure 3. Jet erosion tests on a soil where the cohesion model at particle contacts presents a small yield value under tension (top), under shear (middle), and under bending (bottom), respectively.

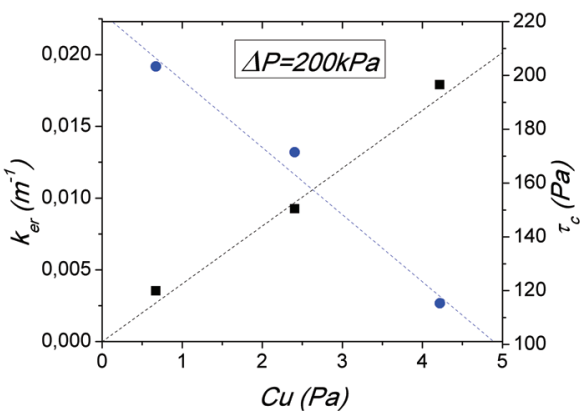

Figure 4. Linear correlations between the erodibility parameters $\tau_{c}$ (black squares) and $k_{e r}$ (blue circles) of a kinetic sand, deduced from the classical interpretation model with a $2 \mathrm{D}$ pressurised-air jet test (differential pressure $\Delta P=200 P a$ ), and the undrained cohesion $\mathrm{Cu}$, obtained from a fall-cone test.

test and hole erosion test, were used but sometimes with specific adaptations (fluid, geometry, optical techniques) enabling direct visualization of the local process. By way of example, Figure 2 shows a jet erosion test on a magic sand where the geometry was restricted to 2D for observation purpose. Some rather usual mechanical tests were carried out with our artificial materials, as fall-cone test, while several other ones were specifically developed for instance to measure tensile strength both at contact and sample scales [7].

\section{Some preliminary results}

Quite an extensive study of our first model material (i.e. glass beads bonded by solid bridges), subjected to erosion by impinging of an immersed jet, has already been carried out by part of the present authors and will be presented elsewhere [7]. However, several recent and original results can be presented, even if they are still preliminary 


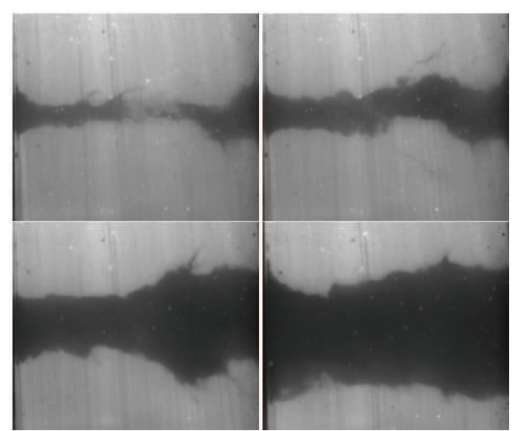

Figure 5. Time evolution of an initially cylindrical pipe in a transparent synthetic clay sample during a Hole Erosion Test with water flow from left to right. The flow rate is progressively increased and the pictures correspond (from left to right and top to bottom) to successive times $t=94 \mathrm{~s}, t=200 \mathrm{~s}, t=350 \mathrm{~s}$, and $t=800 \mathrm{~s}$. The dimension of the pictures is $70 \mathrm{~mm} \times 58 \mathrm{~mm}$.

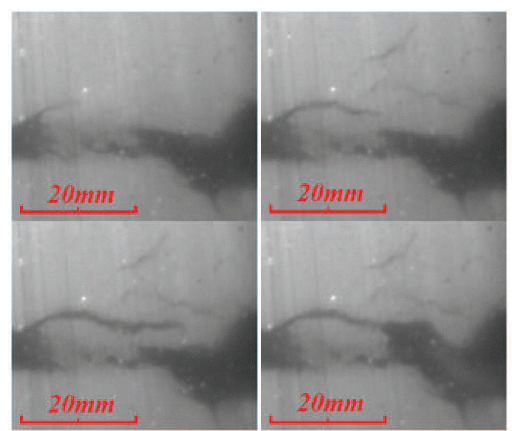

Figure 6. Close-up from the previous test (Fig. 5) where a crack propagation is clearly identified above the pipe and induces the final pull-out of a large aggregate.

at this stage. To start with, our first type of model material has also been studied in a jet erosion configuration but this time by means of a numerical DEM-LBM modeling as previously mentioned. More precisely, the influence of the cohesion model at particle contacts was tested through deliberately exaggerated soil's behaviours by imposing a very weak value of the solid bond yield alternatively under tension, shear and bending loading. As can be seen on Figure 3, widely different erosion processes are observed in the three distinct cases: (i) almost usual grainby-grain erosion, (ii) remote debonding to a rather long extent within the sample, (iii) in-depth fracturing creating large aggregates.

Another example deals with the same kind of jet erosion test but performed this time on one of our second model materials, namely kinetic sand, and with pressurised air as eroding fluid. Changing the initial density of the kinetic sand allows to control quite accurately the undrained cohesion $C u$ estimated via a cone-fall test (by calibration from penetration depth of a falling cone, released from a given height, within a soil's specimen). During a jet test, a classical fitting procedure of the scour crater depth versus time using Eq. 1 provides the erodibility parameters $\tau_{c}$ and $k_{e r}$ which, as underlined in Figure 4, are nicely and almost linearly correlated to $C u$.
Finally, a last preliminary study has focused on hole erosion test performed on a Laponite synthetic clay, our third model material of cohesive soils, using the classical Hole Erosion test (HET) device [1] where a controlled flow is generated along a preformed axial hole within the soil sample to determine a critical value of hydraulic shear stress. Figure 5 gives a general overview of the pipe enlargement versus time while Figure 6 is a close-up where crack initiation and propagation can be observed till a large aggregate is ultimately washed away.

\section{Conclusion}

The starting point of the present contribution was the identified limitations and remaining issues regarding theoretical and experimental state-of-the-art in quantifying erosion of natural soils. It was then proposed to reverse the classical methodology, substituting real soils by artificial materials, in both experimental and numerical perspectives, with the purpose to better control key properties of the materials so as to implement systematic analysis of sensitivity to erosion, including whenever possible direct visualization of the basic local processes of solid matter removal by a fluid flow as well as specifically selected, or developed, mechanical tests. Several examples of such model materials were first described before some encouraging preliminary results were presented and discussed. Much more work is now required to achieve comprehensive results continuing this strategy.

\section{Acknowledgements}

The authors acknowledge a financial support from the region PACA (France) through the APEX-ESCAPE project.

\section{References}

[1] S. Bonelli (Ed.). Erosion in Geomechanics Applied to Dams and Levees. Wiley-ISTE (2013)

[2] E. Partheniades. J. Hydr. Div. ASCE 91(HY1) 105139 (1965)

[3] J.-Y. Delenne, M.S. El Youssouffi, F. Cherblanc, J.C. Bénet. Int. J. Num. Anal. Meth. Geomech. 28 15771594 (2004)

[4] A. Knapen, J. Poesen, G. Govers, G. Gyssels, J. Nachtergael. Earth-Sciences Reviews 80(1-2) 75-109 (2007)

[5] P. Philippe, M. Badiane, Phys. Rev. E. 87(4) 042206 (2013)

[6] P. Cuéllar, P. Philippe, S. Bonelli, N. Benahmed, F. Brunier-Coulin, J. Ngoma, J.-Y. Delenne, F. Radjaï. Proceedings of the 4th International Conference on Particle-based Methods - Fundamentals and Applications, PARTICLES 2015. E. Oñate, M. Bischoff, D.R.J. Owen, P. Wriggers \& T. Zohdi (Eds).

[7] F. Brunier-Coulin, J.-L. Sarrat, P. Cuéllar, P. Philippe. (Accepted for P\&G2017). 